\title{
Sexual Self-Schemas, Sexual Dysfunction, and the Sexual Responses of Women with a History of Childhood Sexual Abuse
}

\author{
Alessandra H. Rellini · Cindy M. Meston
}

Received: 30 June 2009/Revised: 24 August 2010/ Accepted: 24 August 2010/ Published online: 8 December 2010

(C) The Author(s) 2010. This article is published with open access at Springerlink.com

\begin{abstract}
Accumulating evidence points to the mediating effects of sexual self-schemas on the sexual difficulties of women with a history of childhood sexual abuse (CSA). The following study adds to the extant literature by investigating (1) sexual function and (2) sexual satisfaction utilizing validated measures, and reporting on the relationship between sexual self-schemas and physiological (vaginal photoplethysmography), subjective, and affective responses during laboratory exposure to sexual stimuli. In a community sample of women with $(N=48)$ and without $(N=48)$ a history of CSA, we tested (1) the mediation of negative affect on the relation between sexual self-schemas and sexual function/satisfaction, (2) the mediation of negative affect in the relation between CSA and sexual function/satisfaction, and (3) the mediation of sexual self-schemas in the relation between a history of CSA and negative affect prior to sexual stimuli. We found that more Embarrassed/Conservative and less Romantic/Passionate sexual self-schemas predicted negative affect prior to exposure to sexual stimuli which, in turn, predicted levels of sexual satisfaction. The lower sexual satisfaction of CSA survivors was partially mediated by higher reports of negative affect prior to sexual stimuli. However, negative affect prior to sexual stimuli was not mediated by the sexual self-schemas of CSA survivors. Thus, although sexual self-schemas predicted sexual satisfaction, they did not predict variance in negative affect prior to sexual videos experienced by women with a history of CSA.
\end{abstract}

\footnotetext{
A. H. Rellini $(\bowtie)$

Department of Psychology, University of Vermont, John Dewey

Hall, Burlington, VT 05402, USA

e-mail: arellini@uvm.edu

C. M. Meston

Department of Psychology, University of Texas at Austin, Austin, TX, USA
}

Keywords Sexual self-schema $\cdot$ Sexual arousal . Vaginal photoplethysmography $\cdot$ Childhood trauma Sexual abuse $\cdot$ Sexual function

\section{Introduction}

A history of childhood sexual abuse (CSA) is often associated with an array of problems in adulthood, including sexual dysfunction and sexual dissatisfaction (for a review, see Leonard \& Follette, 2002; Loeb et al., 2002). The literature indicates that sexual problems in CSA survivors include low sexual desire, problems becoming sexually aroused, reaching orgasm, vaginismus, dyspareunia, and low sexual satisfaction (Leonard \& Follette, 2002). The focus of the current study was on sexual arousal (including sexual arousal function and sexual responses to erotic videos) and sexual satisfaction. Most information on sexual arousal function in women with a history of CSA relies on descriptive data based on retrospective self-report measures. Only a paucity of studies have investigated the mechanisms of sexual dysfunction and dissatisfaction in this group of women (Rellini, 2008).

Although specific theoretical models for the sexual arousal function of women who experienced CSA are lacking in the literature, it is possible to borrow from cognitive theories that posit representations of the self (self schemas) are implicated in psychopathologies in women who experienced CSA (Putnam, 1990). Broadly, schemas are defined as filters through which people perceive, organize, and understand information relevant to the self. Schemas are used to interpret and control responses to the world by regulating cognitions, affect, and behaviors (e.g., Kihlstrom \& Cantor, 1983; Markus \& Zajonc, 1985), and therefore are of relevance to many cognitive-behavioral treatments. Empirical studies on mood and anxiety disorders have found convincing evidence that schemas play a key role in the 
development and maintenance of psychiatric disorders (Gibb \& Alloy, 2006; Lumley \& Harkness, 2007; Stuewig \& McCloskey, 2005). Empirical and theoretical evidence also supports a clinically meaningful relationship between sexual self-schemas and sexual dysfunction and dissatisfaction (Cyranowski \& Andersen, 1998; Heiman, 2002). Specifically, models of female sexual function suggest an integral role of schemas in sexual dysfunction (Heiman, 2002; Leiblum \& Wiegel, 2002; Wincze \& Carey, 2001). Empirical support for these models comes from findings indicating that women with sexual dysfunction have weaker positive and stronger negative sexual self-schemas compared to women with no sexual dysfunction (Cyranowski \& Andersen, 1998), and from studies showing improvements in sexual arousal after increases in positive sexual self-schemas (Kuffel \& Heiman, 2006). In summary, schemas have been associated with psychopathologies among adult women with a history of CSA, sexual self-schemas are implicated in sexual function and satisfaction, and women who experienced CSA tend to report more sexual dysfunction and sexual dissatisfaction than the population at large. It is, therefore, feasible that sexual schemas play an integral role in the sexual arousal function of women who experienced CSA.

The relationship between sexual self-schemas and sexual arousal function can be best understood within the context provided by the cognitive-behavioral model for the sexual response originally proposed by Barlow (1986). In this model, the core aspect of problems with sexual arousal function is presumed to be anticipatory anxiety, which is the product of negative expectations (e.g., "My partner will think there is something wrong with me because I cannot become aroused"). This state of anxiety increases the physiological arousal in the individual, which facilitates the narrowing of attention towards non-sexually relevant cues. It is this shift in attention away from sexually-relevant cues that reduces the sexual stimulation necessary to activate sexual arousal. Eventually, the vicious cycle-negative expectations-anticipatory anxiety-distraction from sexual cues-causes problems in sexual function and affects sexual satisfaction. For example, a belief that the experience is doomed to fail may be transformed into a belief about the self (e.g., "I am sexually inadequate"). When this occurs, the individual's sexual self-schemas may be modified in a way that maintains the sexual dysfunction since the problem is now perceived as an essential aspect of the self. In women with a history of CSA, early sexual trauma may affect their perception of sex (Barlow, 1986) and also their perception of their sexual self. As a result, these women may be at risk for experiencing negative expectations about sexual encounters which activate negative affect prior to sexual activities and distract the women from processing sexually-relevant cues that are needed for sexual arousal.

In support of the relationship between sexual self-schemas and responses to sexual stimuli, a recent study on cancer survivors reported that positive sexual self-schemas buffered the relationship between sexual satisfaction and depressive symptoms (Carpenter, Andersen, Fowler, \& Maxwell, 2009), a finding that was interpreted as an indication that individuals with negative sexual self-schemas may approach sexual difficulties following gynecological cancer with a negative cognitive style that may exacerbate depressed mood. A greater understanding of not only the relationship between sexual self-schemas and depressive mood but between sexual self-schemas and negative affect prior to sexual activities and the impact of this relationship on sexual responses would be informative for the development of clinical interventions geared towards sexually abused individuals.

The study of sexual self-schemas is limited by the available methodologies for measuring this construct. The most common operationalization of sexual self-schemas adopts 24 traitadjectives (the Sexual Self-Schema Scale; SSSS; Cyranowski \& Andersen, 1998). Relevant to sexual abuse, a recent study that utilized the SSSS reported initial evidence for a mediation effect of a low romantic/passionate sexual self-schema on the relationship between a history of CSA and negative affect during sex with a partner (Meston, Rellini, \& Heiman, 2006). In that study, a sample of 48 CSA participants and 71 women with no prior history of abuse were asked to complete the SSSS and to answer a set of 10 items from the Brief Index of Sexual Function (Taylor, Rosen, \& Leiblum, 1994) and the Brief Sexual Functioning Questionnaire (Reynolds, Frank, \& Thase, 1988). However, the interpretation of these results was limited by the lack of a validated measure of sexual function. Despite these limitations, this was the first study to investigate sexual self-schemas and sexual function in women who experienced CSA.

The primary goal of the present study was to determine the relationship between sexual self-schemas, negative affect, and sexual arousal in women with a history of CSA. The three sexual self-schema subtypes utilized in this study were those identified by Cyranowski and Andersen: Romantic/Passionate, Open/Direct, and Embarrassed/Conservative. Negative affect in expectation of sexual stimuli was measured immediately prior to exposure to sexual stimuli in a laboratory setting, thus reducing potential memory bias. Sexual arousal function was measured with self-reported retrospective questionnaires that addressed sexual arousal function and sexual satisfaction. Previous studies of this nature assessed sexual function with items derived from different scales, some of which used the prior two years as a reference point while others referred to the previous month (Meston et al., 2006). Physiological and psychological measures of sexual arousal were collected in the laboratory during exposure to sexual stimuli. Physiological sexual arousal was measured using vaginal photoplethysmography, and subjective sexual arousal was measured with a lever the participant moved throughout the exposure to sexual stimuli. To the best of our knowledge, only three studies have used vaginal photoplethysmography to assess physiological sexual arousal of women 
who experienced CSA (Laan \& Everaerd, 1995; Rellini \& Meston, 2006; Schacht et al., 2007). In all three studies, women who experienced CSA showed a weaker physiological sexual response in comparison to the control group. Subjective levels of sexual arousal were not found to differ between groups in one study (Rellini \& Meston, 2006). In a second study, the CSA group showed lower levels of subjective sexual arousal than the NSA group (Schacht et al., 2007), but in this study women were asked to maximize and minimize their sexual arousal during exposure to the sexual stimuli and results from both conditions were averaged; thus, it is not possible to tell whether the group differences were due to the instructions or naturally occurring differences between groups.

The present study adds to the literature by utilizing validated questionnaires for sexual function and satisfaction and including both physiological and subjective data collected during laboratory-manipulation of responses to sexual stimuli. Five a priori hypotheses were tested in this study. First, we expected that CSA survivors would show greater negative and lower positive sexual self-schemas compared to women with no history of CSA. Second, based on the model of negative expectancy and sexual arousal dysfunction proposed by Barlow (1986), we hypothesized that individuals with higher Embarrassed/Conservative and lower Open/Direct and Romantic/Passionate sexual selfschemas would show more negative affect prior to sexual stimuli. This, in turn, would explain lower sexual arousal function and satisfaction, and lower physiological and subjective sexual arousal to sexual stimuli in the laboratory. Third, we predicted higher negative affect prior to sexual stimuli, and lower physiological sexual responses, sexual arousal function, and satisfaction among women who experienced CSA as compared to women with no history of CSA. Fourth, we expected that greater negative affect experienced prior to sexual stimuli would mediate the relationship between a history of CSA and lower physiological sexual responses, sexual arousal function, and sexual satisfaction. Fifth, we expected that sexual self-schemas would predict greater negative affect prior to exposure to sexual stimuli among women who experienced CSA.

\section{Method}

\section{Participants}

Women with a history of CSA $(N=53)$ and women with no history of abuse (NSA; $N=50$ ) were recruited from two medium sized towns in the North East and the South West of the United States through advertisements in local newspapers and fliers posted in laundromats, local stores and services, and public bathrooms. The advertisement specified that a research project on sexual health conducted at the local university was looking for both women with and without a history of unwanted sexual experience. For this study, CSA was defined as a sexual encounter in which touching or penetration of the genitals happened before age 16 with someone at least 5 years older (Finkelhor, Hotaling, Lewis, \& Smith, 1989). Women were included in the CSA group if they responded positively to the following questions asked during the phone screening: (1) "Have you experienced a forced or coerced sexual activity prior to age 16?"; (2) "Did this activity occur with a person or persons that were 5 or more years older than you?"; (3) "Did you experience any genital touching or penetration during the sexual activity?" The information was later checked during an open ended question posed towards the end of a face-to-face interview with the first author (AHR). During the face-to-face interview, of the women in the CSA group, five reported a sexual experience that either was not with someone 5 years or older than them $(n=2)$ or that did not include genital penetration or touching $(n=3)$; of the NSA group, two reported a sexually abusive experience after age 16 . These participants were excluded from the study leaving 48 women in the CSA group and 48 women in the NSA group. Of these women, 8 women from the CSA group and 22 from the NSA group were recruited from a rural area in the North East. No significant differences were found between CSA participants recruited from the South West and those from the North East, in severity of childhood trauma as measured by the Child Trauma Questionnaire (see section below for a description of the measure). Also, no significant differences were found between participants in the two sites for physiological sexual responses, subjective sexual responses, and sexual self schemas scores (analyses were computed for CSA and NSA groups separately). No information is available on the number of people that were excluded from this study specifically for eligibility criteria because our laboratories automatically screen for all active studies; thus, some women may have qualified for this study but were scheduled for a different study.

The following inclusion criteria were met by all women in the study: age between 25 and 35 years, fluent in English, predominantly or exclusively heterosexual, and currently sexually active with a partner or partners. The exclusion criteria for both the CSA and NSA groups included having experienced a traumatic event in the previous three months, being currently involved in an abusive relationship, taking antidepressants or beta blockers for less than three months, reporting sexual side effects from any medication, and reporting seeing or hearing things that other people could not see or hear during the previous 6 months. Also, reports of medications taken to control psychoses and reports of hospitalization because of a psychotic episode were considered exclusion criteria. If participants received medications known to affect sexual function (e.g., antidepressants), they were included in the study under the condition that they had been stabilized as per the medication type and dosage for at least three months prior to the study and reported no changes after taking the medication. In the NSA 
group, one woman was taking venlafaxine, while in the CSA group one woman was taking quetiapine, two were taking bupropion (one of them was also taking fluoxetin), and one was taking escitalopran. Recreational drugs were reported in the sample with marijuana being used 4 days or more prior to the study by eight women in the CSA group and two in the NSA group. Stimulants (cocaine, methylphenidate, and speed) were reported in the week prior to the study by three of the women in the CSA group. Pain killers taken for recreational purposes were reported by one woman in the NSA group and two women in the CSA group. Women did not qualify for the NSA group if they reported any sexual encounter before the age of 16 with someone 5 years or older or if they had ever had a sexual activity against their will. Also, women did not qualify for the NSA group if they reported a history of physical abuse or neglect during childhood.

Participants self-identified as coming from a variety of ethnic backgrounds as illustrated in Table 1, with 4 and 13\% individuals from the NSA and CSA groups, respectively, identifying with more than one ethnic group. The CSA group was older than the NSA group, $t(94)=3.47, p<.01$. No significant statistical differences were observed between the two groups on reported household income, $t(94)=1.37$, type of relationship (dating vs.

Table 1 Demographics for CSA (childhood sexual abuse) and NSA (no sexual abuse) groups

\begin{tabular}{|c|c|c|c|c|c|c|}
\hline & \multicolumn{3}{|l|}{ NSA } & & \multicolumn{2}{|l|}{ CSA } \\
\hline & $M$ & & $S D$ & & $M$ & $S D$ \\
\hline Age (in years) & 25.7 & & 4.91 & & 29.7 & 6.12 \\
\hline Kinsey scale & 0.5 & & 0.75 & & 0.8 & 0.91 \\
\hline \multicolumn{7}{|c|}{ Childhood Trauma Questionnaire } \\
\hline Physical abuse & 6.7 & & 2.74 & & 11.3 & 5.59 \\
\hline Sexual abuse & 5.2 & & 0.94 & & 15.8 & 7.14 \\
\hline Emotional abuse & 9.6 & & 4.25 & & 15.5 & 5.44 \\
\hline Emotional neglect & 7.9 & & 2.60 & & 12.2 & 4.77 \\
\hline \multirow[t]{2}{*}{ Physical neglect } & 6.5 & & 1.52 & & 11.0 & 4.32 \\
\hline & \multicolumn{3}{|c|}{$n$} & $\%$ & $n$ & $\%$ \\
\hline \multicolumn{2}{|l|}{ Relationship: dating } & 11 & & 23 & 19 & 39 \\
\hline \multicolumn{2}{|l|}{ Education: college or higher } & 24 & & 53 & 28 & 62 \\
\hline \multicolumn{2}{|l|}{ Income: $<\$ 50,000$} & 37 & & 78 & 44 & 91 \\
\hline \multicolumn{7}{|l|}{ Ethnicity } \\
\hline \multicolumn{2}{|l|}{ Hispanic } & 6 & & 13 & 5 & 10 \\
\hline \multicolumn{2}{|l|}{ Caucasian } & 39 & & 81 & 32 & 67 \\
\hline \multicolumn{2}{|l|}{ Other } & 3 & & 6 & 11 & 23 \\
\hline
\end{tabular}

Note: Kinsey scale: measure of sexual orientation, absolute range $=0-6$. Childhood Trauma Questionnaire, absolute ranges $=5-25$ for each factor. Ethnicity: Other = Asian Americans, African Americans, and American Indians in a relationship or married), $\chi^{2}(1)=2.38$, or education, $\chi^{2}(1)<$ 1 (for demographics descriptive statistics, see Table 1).

\section{Measures}

\section{Laboratory Measures: Physiological Sexual Arousal}

Measures of changes in physiological sexual arousal induced by sexual stimuli were assessed using vaginal photoplethysmography (Sintchak \& Geer, 1975), a methodology that utilizes vaginal pulse amplitude (VPA) as an index of physiological sexual arousal. Vaginal photoplethysmography consists of a vaginal probe the shape and size of a tampon that the participant inserts privately. A placement bar standardizes the position and the depth of the probe. The sinusoidal pulse signal was sampled 80 times/s with a Biopac system (Santa Barbara ${ }^{\circledR}$ ). The amplitude of each pulse wave (trough to peak vertical distance) was recorded in millivolts $(\mathrm{mV})$ throughout the exposure to a nonsexual video ( $3 \mathrm{~min}$ ) and a sexual video $(10 \mathrm{~min})$. The sexual video was an edited version of a video directed by Candida Royalle, made by women for a female audience and therefore expected to be more sensitive to women's preferences. The video depicted $3 \mathrm{~min}$ of foreplay, $3 \mathrm{~min}$ of oral sex, and $4 \mathrm{~min}$ of vaginal intercourse between a heterosexual couple. In accordance with previous studies of this nature (e.g., Rellini \& Meston, 2006), artifacts caused by movement or contractions of the pelvic muscles were deleted following visual inspection of the data. Percentage of increase in VPA was calculated by computing the difference between the average VPA during the last 30 consecutive seconds of the non-sexual video with the minimum amplitude and the average VPA during the $30 \mathrm{~s}$ of maximum arousal during the sexual video. The final VPA score was computed utilizing the following logarithm:

$$
\begin{aligned}
\mathrm{VPA}= & {\left[\left(\mathrm{MeanVPA}_{\text {SexualVideo }}-\text { MeanVPA }_{\text {NonSexualVideo })}\right.\right.} \\
& \times 100] / \text { MeanVPA }_{\text {NonSexualVideo }}
\end{aligned}
$$

\section{Laboratory Measures: Subjective Sexual Arousal}

Subjective ratings of the participants' sexual arousal (SSA) during the video sequence were measured with a device termed an "arousometer." The arousometer consists of a computer optical mouse (Intellimouse by Microsoft ${ }^{\circledR}$ ) mounted on a wooden track divided into equally spaced intervals from 0 to 7. Participants were asked to move the mouse continuously throughout the non-sexual and sexual videos to indicate how sexually aroused they felt. Previous studies have shown this device to be a reliable and valid indicator of sexual arousal in women (e.g., Rellini, McCall, Randall, \& Meston, 2005). SSA scores were first transformed into a $0-100$ scale where 0 on the arousometer corresponded to a score of 0 , and a 7 on the arousometer corresponded to a score of 100. Subsequently, the 
average of SSA was computed during the exposure to the neutral and erotic videos. All participants reported 0.00 SSA during the non-sexual video.

\section{Laboratory Measures: Affective Responses}

The Positive and Negative Affect Schedule (Watson, Clark, \& Tellegen, 1988) assesses two global dimensions of affect: negative and positive affect. A large body of literature supports the psychometric properties of the PANAS (see Watson, 2000). In the version of the PANAS used here, participants were instructed to complete the scale based on how they felt immediately prior to watching the video sequence. Scores for negative affect were used as an indication of affect in anticipation of the video (NA-stimuli).

\section{Sexual Arousal Function}

Participants completed the Female Sexual Function Index, a widely used questionnaire that differentiates between women with and without female sexual arousal disorder (Rosen et al., 2000), and between sexually healthy women and women with female orgasmic disorder and hypoactive sexual desire disorder (Meston, 2003). The scale is divided into six domains, one of which is sexual arousal. Response options ranged from 0 to 6 , with higher scores indicating higher functioning. As reported by Rosen et al. (2000), all subscales have acceptable internal consistency (Cronbach's $\alpha=0.95$ ), inter-item reliability (Cronbach's $\alpha=0.82-0.92$ ), and test-retest reliabilities during a 4-week interval (Pearson's $r=.79-.88$ ). Divergent validity has been established using the Locke-Wallace Marital Adjustment Scale (Pearson's $r=.53$ for women with female sexual arousal dysfunction, Pearson's $r=.22$ for sexually functional women). In this study, we utilized the arousal domain score (Arousal-function).

\section{Sexual Satisfaction}

An important aspect of female sexuality is sexual satisfaction; this construct was measured with the Sexual Satisfaction Scale-Women (SSS-W; Meston \& Trapnell, 2005), a 30-item questionnaire that operationalizes satisfaction into five separate domains supported by factor analysis: ease and comfort discussing sexual and emotional issues (Communication), compatibility between partners in terms of sexual beliefs, preferences, desires, and attraction (Compatibility), contentment with emotional and sexual aspects of the relationship (Contentment), personal distress concerning sexual problems (Personal Distress), and distress regarding the impact of their sexual problems on their partners and relationships at large (Relational Distress). The SSS-W domains have acceptable internal consistency (Cronbach's $\alpha \geq 0.74$ ) and test-retest reliability ( $r=.58-.79)$. The SSS-W total score reliably differentiates between women with and without sexual dysfunction (Meston \& Trapnell, 2005). In the present study, the total score (Satisfaction) was utilized.

\section{Sexual Self-Schema}

The Sexual Self-Schema Scale (SSSS; Andersen \& Cyranowski, 1994) was used as a measure of one's perception of the self as a sexual being. This scale consists of 50 trait-adjectives (26 scored and 24 fillers) that participants rate on a scale from 0 (not at all descriptive of me) to 6 (very much descriptive of me). The items were subdivided into three statistically-determined domains: two positive schemas (Open/Direct, and Passionate/Romantic), and one negative schema (Embarrassed/ Conservative). Contributing to the Romantic/Passionate dimension were 10 adjectives: romantic, passionate, unromantic, warm, loving, feeling, sympathetic, arousable, stimulating, and revealing. The Open/Direct dimension was comprised of nine adjectives: direct, straightforward, frank, outspoken, broad-minded, experienced, casual, open-minded, and uninhibited; and the Embarrassed/Conservative aspect was comprised of seven adjectives: cautious, timid, self-conscious, prudent, embarrassed, conservative, and inexperienced. These three schemas have adequate construct validity as evidenced by convergent and discriminant validity with romantic attachment, relationship satisfaction, sexual esteem, sexual desire, and erotophobia (Cyranowski \& Andersen, 1998). Andersen and Cyranowski (1994) reported an internal consistency of $\alpha=0.82$ and test-retest reliability (2-week interval), $r=.91$, for the total score. In the present study, the inter-item correlations were Cronbach's $\alpha \mathrm{s}=0.82,0.83$, and 0.68 for the Romantic/ Passionate, Open/Direct, and the Embarrassed/Conservative domains, respectively. Given that previous studies found differences between the three sexual self-schemas of women who experienced CSA, this study utilized scores for each of the three separate schemas.

\section{Child Sexual Abuse}

The Childhood Trauma Questionnaire (CTQ) is a 60-item questionnaire that reliably measures history of CSA, physical abuse, emotional abuse, physical neglect, and emotional neglect (Bernstein \& Fink, 1998). Internal consistency estimates are high for each of the four factors (Cronbach's $\alpha \mathrm{s}=0.79-0.94$ ) and the total scale $(\alpha=0.95)$. Test-retest reliabilities were good for the individual factors $(0.80-0.83)$ and the total scale $(0.88)$ (Bernstein \& Fink, 1998). Convergent validity has been established using a structured interview (Bernstein et al., 1994). Positive responses (from "rarely true" to "always true") to items on sexual abuse and molestation were used to confirm a history of CSA also reported during the phone screening and a semistandardized interview designed to assess for sexual abuse history. 


\section{Procedure}

After a brief phone screening that assessed inclusion and exclusion criteria, women were invited for one 2 -h session at the laboratory. During this session, they completed a sexual psychophysiological assessment that consisted of watching a 3-min video on a travelogue about Russia (non-sexual video) followed by a 10-min sexual video showing scenes of a heterosexual couple engaging in explicit sexual behaviors. The video has previously been shown to increase both physiological and subjective sexual arousal in women with and without a history of CSA (Rellini \& Meston, 2006). Before the video started, participants were left alone in the private, internally locked room equipped with a recliner chair, a TV, and an intercom system. Participants were instructed by a female experimenter to insert the vaginal probe and, after a 10 min of resting (habituation period), they completed the pre-video version of the PANAS. While watching the video sequence, participants used the arousometer to indicate their level of subjective sexual arousal. Immediately following the sexual video, participants completed a variety of questionnaires, including the FSFI, the SSS-W, and the CTQ. An exit interview was used to confirm CSA status and to collect more information on the type of abuse and other psychiatric variables not reported here. Participants were debriefed and compensated $\$ 60$. All study procedures were approved by the Humans Subjects Ethics Committee of the University of Vermont and the University of Texas at Austin.

\section{Statistical Analyses}

Mediation analyses were computed for negative affect prior to sexual stimuli (mediator) in the relationship between schemas (independent variable) and (1) Arousal-function, (2) Satisfaction, (3) VPA, and (4) SSA (dependent variables). Before testing the mediation model between the three sexual selfschemas and the sexual variables, we computed zero-order correlations to test for the presence of a significant relationship between negative affect and the four target variables (Arousalfunction, Satisfaction, VPA, and SSA). When the correlation coefficient was significant, the variable was included in the mediation model. Mediation was tested using the guidelines provided by MacKinnon and Dwyer (1993) and MacKinnon, Fairchild, and Fritz (2007): we tested the effects of the independent variable to the mediator (path $\alpha$ ) and the effect of the mediator to the dependent variable when the independent variable was taken into account (path $\beta$ ). This procedure is a variation on the Sobel (1982) test that accounts for the nonnormal distribution of the $\alpha \beta$ path. We performed bootstrapping to ensure the robustness of the results. According to guidelines mentioned above (MacKinnon et al., 2007), a full mediation is assumed if the relationship between the independent and the dependent variables is no longer significant when the mediator is included in the model, while a partial mediation is assumed if the relationship between independent and dependent variables remains significant but is substantially reduced when the mediator is included in the model. A partial mediation provides indication that other mechanisms may be at play other than one tested in the model.

To test the third hypothesis that the CSA group would differ from the NSA group in Arousal-function, Satisfaction, NAstimuli, VPA, and SSA, we computed a series of ANCOVAs. To identify the potential covariates to include in the model, a series of $t$ tests between CSA and NSA were computed for all main demographic variables (age, sexual orientation, ethnicity, relationship status), with categorical variables (i.e., ethnicity and relationship status) dummy coded.

A Sobel test for mediation similar to the one described above was utilized to assess the effect of NA-stimuli (mediator) on the relationship between group (IV: $\mathrm{NSA}=0$; $\mathrm{CSA}=$ 1) and Arousal-function and Satisfaction (DVs). A separate Sobel test was computed for each DV. The group was entered as categorical variables and all participants were included in all the analyses.

\section{Results}

Group Differences (CSA vs. NSA) in Sexual Function and Sexual Responses to Sexual Stimuli

Since we found a significant age difference between CSA and the NSA groups, age was used as covariate in the following analyses that compared the two groups. Women in the CSA group reported significantly less Arousal-function, $F(1,83)=$ $5.82, p=.05$, less Satisfaction, $F(1,83)=11.80, p<.01$, and more NA-stimuli, $F(1,83)=4.64, p<.05$, than women in the NSA group. These results indicate that a history of CSA was associated with lower sexual arousal function, sexual dissatisfaction, and greater negative affect prior to exposure to sexual stimuli. SSA did not differ between the two groups, $F(1$, $77)=1.64$ and there was no significant group difference in VPA, $F(1,75)<1$ (Table 2$)$.

\section{Mediation of Negative Affect in the Relationship Between} Schemas and Sexual Function/Responses

NA-stimuli was significantly correlated with Arousal-function, $r(94)=-.22, p<.05$, and with Satisfaction, $r(94)=$ $-.31, p<.01$. VPA and SSA were not significantly correlated with NA-stimuli, $r(94)=.03$, and $r(94)=.14$, respectively. Given these results, mediation analyses were computed only for Arousal-function and Satisfaction (dependent variables).

In the first mediation analysis, path $\alpha$ corresponded to the relationship between Romantic/Passionate (independent variable) and NA-stimuli (mediator); path $\beta$ corresponded to the 
Table 2 Group differences for sexual function, sexual responses, and sexual self-schemas

\begin{tabular}{|c|c|c|c|c|c|}
\hline & \multicolumn{2}{|l|}{ NSA } & \multicolumn{2}{|l|}{ CSA } & \multirow[t]{2}{*}{$F$} \\
\hline & $M$ & $S D$ & $M$ & $S D$ & \\
\hline Arousal-function & 4.9 & 0.86 & 4.4 & 1.04 & $5.82 *$ \\
\hline Satisfaction & 118.9 & 22.39 & 101.2 & 24.32 & $11.80^{* * *}$ \\
\hline VPA & 11.7 & 24.64 & 5.0 & 12.51 & $<1$ \\
\hline SSA & 54.0 & 31.23 & 69.8 & 30.60 & 1.64 \\
\hline \multicolumn{6}{|l|}{ SSSS } \\
\hline Romantic/Passionate & 46.2 & 7.59 & 45.3 & 8.87 & $<1$ \\
\hline Open/Direct & 37.4 & 8.00 & 39.8 & 8.01 & 2.89 \\
\hline Embarrassed/Conservative & 16.6 & 5.94 & 15.8 & 6.32 & $<1$ \\
\hline
\end{tabular}

Note: The $F$ statistics corresponds to ANCOVAs where age was used as covariate. Arousal-function $=$ FSFI arousal domain, absolute range $=$ $0-6$, Satisfaction $=$ SSS-W total score, absolute range $=30-150, \mathrm{VPA}=$ vaginal pulse amplitude; $S S A=$ subjective sexual arousal measured with the Arousometer, absolute range $=0-100 ;$ SSSS $=$ score for the subfactors of the Sexual Self Schema Scale, absolute ranges for: Romantic/Passionate $=0-60$, Open/Direct $=0-54$, and Embarrassed $/$ Conservative $=0-42$ $* p<.05 ; * * * p<.001$

relationship between NA-stimuli (mediator) and Satisfaction (dependent variable) when taking into consideration Romantic/Passionate, and path c' was the direct effect of Romantic/ Passionate on Satisfaction when taking paths $\alpha$ and $\beta$ into account. Results indicated that NA-stimuli partially mediated the relationship between Romantic/Passionate and Satisfaction (lower limit $=0.02$, upper limit $=0.43$ ). Partial mediation was established because the relationship between DV and IV remained significant although substantially reduced by the mediator (Fig. 1, Panel A). The same test was computed utilizing Embarrassed/Conservative as IV. Results showed that the $95 \%$ confidence interval of the indirect path $(\alpha \beta)$ did not overlap with zero (lower limit $=-0.96$, upper limit $=-0.04$ ), supporting our first hypothesis that negative affect prior to sexual stimuli (negative affect) fully mediated the relationship between Embarrassed/Conservative and Satisfaction (Fig. 1, Panel B). These results indicated that higher scores on the Embarrassed/Conservative sexual self-schema explained higher negative affect prior to sexual stimuli and this explained lower sexual satisfaction. Conversely, higher scores on the Romantic/Passionate sexual self-schema explained lower negative affect prior to sexual stimuli which explained higher levels of sexual satisfaction.

Mediation was not detected for the relationship between Open/ Direct and Satisfaction (lower limit $=-0.02$, upper limit $=0.48$ ). Also, no significant mediation was observed for NA-stimuli in the relationship between the three sexual self-schemas and Arousal-function (results not shown in the figure).

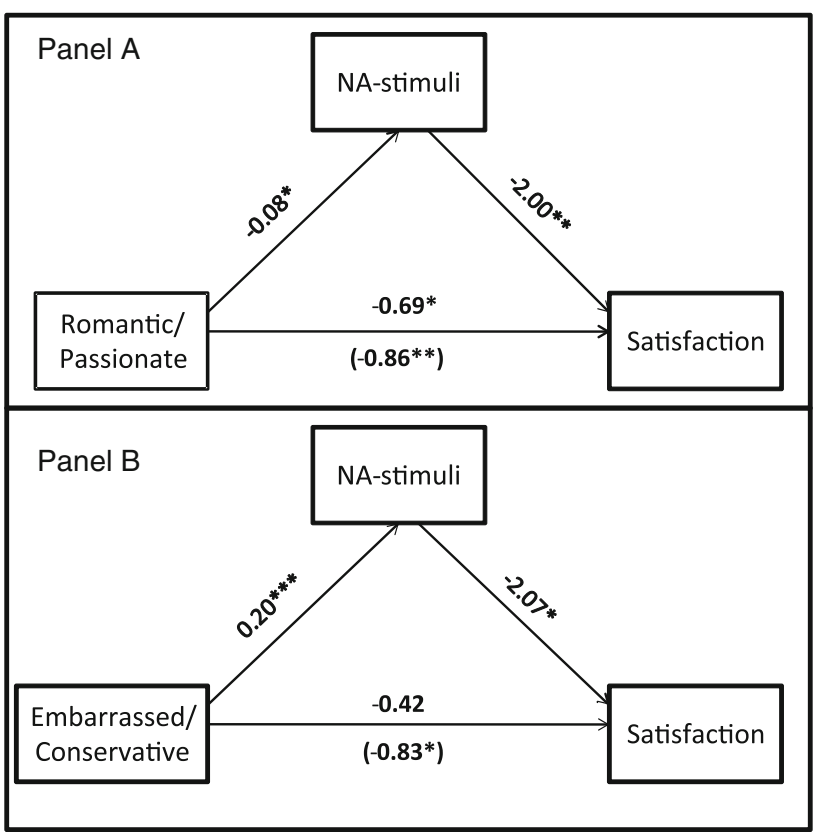

Fig. 1 Illustration of the Sobel coefficients for the mediation of negative affect prior to sexual stimuli (NA-stimuli) on the relationship between Romantic/Passionate sexual self schemas and sexual satisfaction (Satisfaction) in Panel A. In Panel B, illustration of the Sobel coefficients for the mediation of negative affect prior to sexual stimuli (NA-stimuli) on the relationship between Embarrassed/Conservative sexual self-schemas and sexual satisfaction (Satisfaction). Numbers in parentheses correspond to coefficients for the total effect (path c). ${ }^{*} p<.05 ; * * p<.01$; $* * * p<.001$

Mediation of Negative Affect in the Relationship Between CSA and Sexual Function/Satisfaction

Since group differences were not observed for VPA and SSA, we computed mediation tests only for the relationships between group and Arousal-function (Fig. 2, Panel A), and between group and Satisfaction, in all cases using age as a covariate (Fig. 2, Panel B). The same Sobel procedure described above was utilized to assess mediation of NA-stimuli in the relationship between group (CSA, NSA) and Arousal-function and Satisfaction (DVs). Group was associated with NA-stimuli, $z=1.60, p<.05$, and NA-stimuli was associated with Arousalfunction, $z=-0.06, p<.05$. There was also a significant direct path between group and Arousal-function, $z=-0.41$, but the Sobel test did not support mediation (lower limit $=-0.43$, upper limit $=0.003$ ) (Fig. 2, Panel A).

A Sobel test supported the mediation model of NA-stimuli (mediator) in the relationship between the independent variable (group: $\mathrm{CSA}=1, \mathrm{NSA}=0$ ) and the dependent variable (Satisfaction), when controlling for age (Fig. 2, Panel B). These results indicated that the $95 \%$ confidence interval of the indirect path did not overlap with zero (lower limit $=-8.67$, upper limit $=-0.41$ ), suggesting that negative affect prior to exposure 


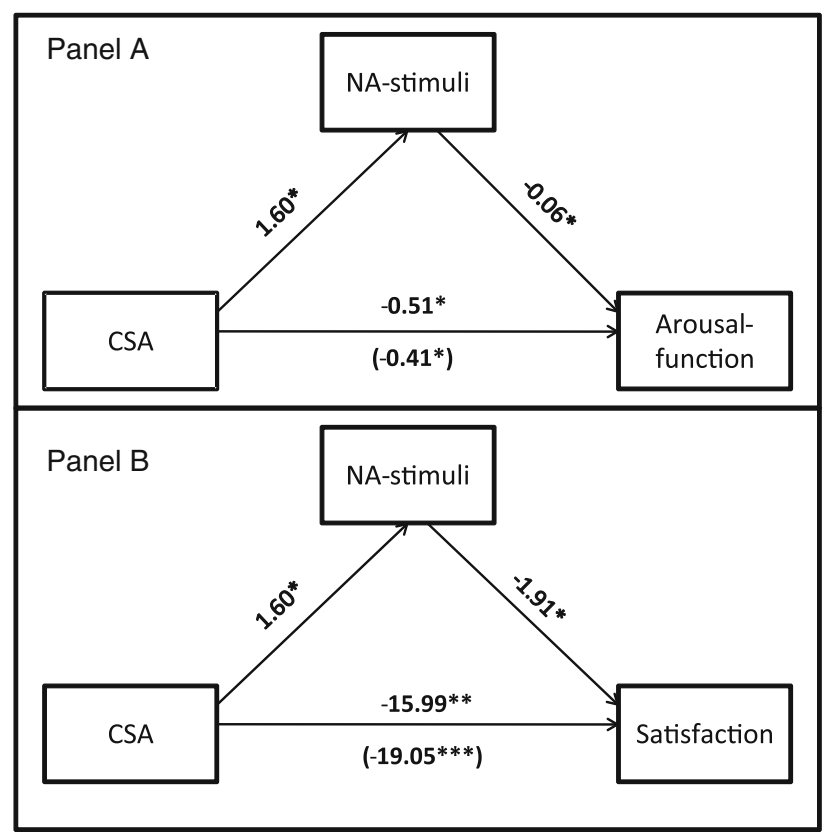

Fig. 2 Illustration of the Sobel coefficients for the mediation of negative affect prior to sexual stimuli (NA-stimuli) on the relationship between CSA and sexual arousal function (Arousal-function), in Panel A, and sexual satisfaction (Satisfaction), in Panel B. Numbers in parenthesis correspond to coefficients for the total effect (path c). $* p<.05 ; * * p<.01$; $* * * p<.001$

to sexual stimuli mediated the relationship between CSA and sexual satisfaction.

\section{Mediation of Sexual Self-Schemas in the Relationship Between CSA and Negative Affect}

Our fourth hypothesis, based on trauma and sexual function models, predicted that sexual self-schemas would explain the higher negative affect observed in women who experienced CSA. Since NA-stimuli mediated the relationship of Romantic/ Passionate and Embarrassed/Conservative with Satisfaction, only Romantic/Passionate and Embarrassed/Conservative (not Open/Direct) were used as mediators between group $(\mathrm{CSA}=1$, NSA $=0$ ) and NA-stimuli. Results from the Sobel test showed that only Embarrassed/Conservative was significantly associated with NA-stimuli, $z=4.00, p<.001$. Both the total and the indirect effect of group on NA-stimuli were significant, $z=$ $2.15, p<.05$, and $z=2.14, p<.05$, respectively (Fig. 3). The Sobel test of mediation did not support the mediation in that CSA was not associated with either Romantic/Passionate, $z=-0.70 ;$ or Embarrassed/Conservative, $z=0.32$.

\section{Discussion}

This study found partial support for the hypothesis that sexual self-schemas and negative affect prior to sexual stimuli

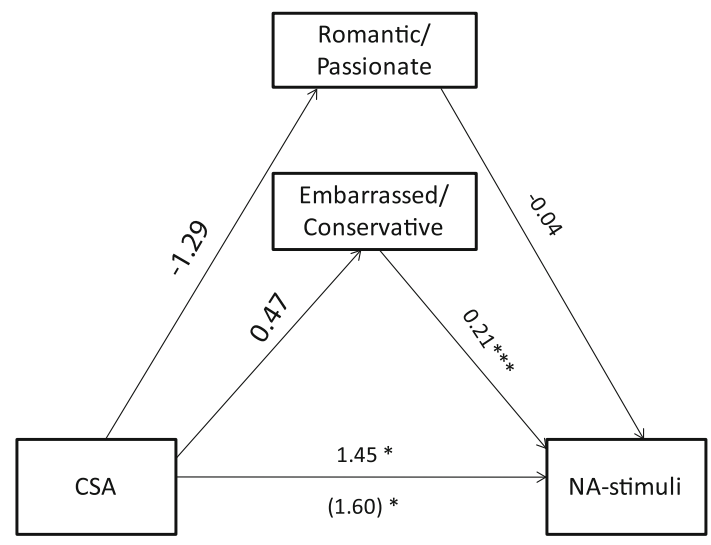

Fig. 3 Illustration of the Sobel coefficients for the mediation of Romantic/Passionate and Embarrassed/Conservative schemas in the relationship between CSA and negative affect prior to sexual stimuli (NAstimuli). Numbers in parenthesis correspond to coefficients for the total effect (path c). *p $<.05 ; * * p<.01 ; * * * p<.001$

partially explained the lower sexual arousal function and sexual satisfaction of women who experienced CSA compared to women with no history of abuse. It is worth noting that between group differences were greater for sexual satisfaction, where the CSA fell within one $S D$ from the average score for a clinical sample with female sexual arousal dysfunction. While for sexual satisfaction, the CSA group scored only slightly lower than the NSA group. These between group differences parallel findings from previous studies indicating that sexual satisfaction, more than functioning, is more markedly lower in women with a history of CSA when compared to a group of women with no history of abuse (Rellini \& Meston, 2007). For all women, data supported the hypothesis that negative affect prior to sexual stimuli mediated the relationship between negative sexual selfschemas (i.e., Embarrassed/Conservative) and lower sexual satisfaction, suggesting that more negative sexual self-schemas could lead to negative affect prior to sexual experiences, thereby reducing sexual satisfaction.

Negative affect prior to sexual stimuli was also associated with lower sexual arousal function, which supports Barlow's (1986) model of sexual arousal function. However, negative affect prior to sexual stimuli did not predict either subjective or physiological sexual responses measured in the laboratory. Barlow's model of sexual arousal function has been tested in men and laboratory studies have found that negative expectations and negative affect prior to sexual stimuli predicted lower physiological sexual arousal in men (Wiegel, Scepkowski, $\&$ Barlow, 2007). Studies testing Barlow's model in women have yielded mixed results, with several studies reporting a positive association between anxiety and genital sexual responses in women (Beggs, Calhoun, \& Wolchik, 1987; Laan, Everaerd, van Aanhold, \& Rebel, 1993; Palace \& Gorzalka, 1990; Wincze, Hoon, \& Hoon, 1977). Indeed a meta-analysis found that negative affect was among the best predictors of sexual arousal, 
although all predictors together explained only $9 \%$ of variance in genital sexual responses (Laan \& Everaerd, 1995). In this light, our results were not unusual and may point to the inadequacy of either Barlow's model to explain the sexual arousal problems of women or the inadequacy of the sexual psychophysiological data to parallel the sexual responses of these women during sexual activities.

The observed mediation of negative affect in the relationship between sexual self-schemas and sexual satisfaction can be best understood within the cognitive-behavioral tradition that assumes a circular association among cognition, affect, and behavior. According to the cognitive-behavioral conceptualization of psychopathology introduced by Beck (1996), cognitions affect our affective responses to situations and affect influences behavior, which then shapes cognition. Prior research has provided support for the relationship between behavior (difficulties becoming aroused and reaching orgasm) and cognition (i.e., distracting cognitive thoughts) (e.g., Beck, Barlow, Sakheim, \& Abrahamson, 1987; Dove \& Weiderman, 2000; Elliot \& O’Donohue, 1997; Nobre \& Pinto-Gouveia, 2008). Our findings add to this knowledge by providing evidence that emotional responses (negative affect prior to sexual stimuli) are partially responsible for the relationship between cognitions (i.e., sexual self-schemas) and sexual satisfaction. Other studies have also highlighted the importance of negative affect and sexual function (e.g., Beck \& Barlow, 1986; Heiman, 1980; Heiman \& Rowland, 1983; Koukounas \& McCabe, 2001; Meisler \& Carey, 1991; Mitchell, DiBartolo, Brown, \& Barlow, 1998); however, the present study provided a unique contribution to the literature since (1) negative affect was measured in the CSA group, (2) it was measured immediately prior to sexual stimuli and thus provided a more reliable measure of anticipatory affect as compared to recounts of previous sexual experiences, and (3) it utilized a statistical methodology that has been recognized as appropriate to test mediation effects.

In agreement with our second hypothesis, we found that women with a history of CSA had higher negative affect prior to sexual stimuli, and they also scored lower in self-reported measures of sexual arousal function and sexual satisfaction. The third hypothesis was partially supported in that negative affect prior to sexual stimuli mediated the relationship between a history of CSA and sexual satisfaction, but did not explain the lower sexual arousal function in the CSA group, which was independently related to negative affect. The observed mediation of negative affect prior to sexual stimuli in the relationship between CSA and sexual satisfaction further highlights the relevance of the cognitive-behavioral model to better understand the sexual difficulties of women who experienced CSA.

In contrast to what we would expect from the literature on self-schemas (Markus, 1990; Meston et al., 2006), we did not find evidence that more negative sexual self-schemas in the CSA group, nor we found evidence for a relationship between schemas and the higher negative affect prior to sexual stimuli reported by the CSA group compared to the NSA group. It is possible that the schemas measured by the SSSS did not fully capture the schemas involved in the negative affect relevant to sexual stimuli among women in the CSA group. Indeed, in a critical review of the SSSS (Hill, 2007) it was noted that a principal component analysis revealed that only a small percentage $(38 \%)$ of the variance in items' scores was explained by the three schemas. Alternatively, it may be that the schemas that activate negative affect prior to sexual stimuli are not specifically sexual per se but, rather, are related to other aspects of the self.

Inconsistent with our hypotheses, we did not find evidence that negative affect mediated the relationship between sexual self-schemas and sexual arousal function or laboratory indices of physiological and subjective sexual arousal. It is feasible that the sexual difficulties experienced by women with a history of CSA may not be relevant to sexual arousal. For example, the sexual self-schemas of women exposed to CSA may affect the association between distress and sexuality because of problems with sexual compulsion, inability to feel intimate or to let go during sex, or other problems related to risky sexual behaviors, while their sexual arousal function may be intact. In support of this hypothesis, a prior study (Rellini \& Meston, 2007) has also proposed that the low sexual arousal function of women exposed to CSA may not be completely captured by the definition of sexual dysfunction provided by the DSM-IVTR (APA, 2000).

Also inconsistent with hypotheses, the CSA group did not have lower physiological sexual responses as compared to the NSA group. This is different from previous studies reporting group differences (e.g., Laan \& Everaerd, 1995; Rellini \& Meston, 2006). The study by Laan and Everaerd (1995) only observed a small correlation between CSA history and VPA. Also, CSA was only one of many variables included in their analyses, so their finding cannot be directly compared to the results showed in this study. Methodological differences, however, cannot be used to explain the difference between these results and the findings reported by Rellini and Meston (2006) since the procedure, the measures, the recruitment strategies, and the research team were identical. The only difference in methodology between the two studies was the recruitment of participants from the Southwest for the first study and from both the Southwest and the Northeast in this study. However, we did not find significant differences in demographics between these two groups. Data on depression and posttraumatic stress disorder symptoms would be able to inform us on the potential differences in the severity of the impact of the sexual abuse on psychological functioning but unfortunately we did not collect such data in both studies. A third study on sexual psychophysiological responses in women with a history of CSA also found a lower genital sexual response in women in the CSA group as compared to women in the NSA group, but only when women were instructed to maximize their physiological sexual arousal 
(Schacht et al., 2007). This study did not use a basic condition when participants were not given instructions to alter their physiological sexual arousal; therefore, the results of the two studies cannot be directly compared.

Several limitations of this study are worth noting. This study was mostly descriptive since the two groups selected from the population were compared on a number of outcome and mediator variables. Future studies on mechanisms of sexual responses of women with a history of CSA may benefit from an experimental design that utilizes within-group conditions. Also, in this study, we did not assess sexual revictimization. Women with a history of CSA are 2.6 times at higher risk for future sexual abuse during adulthood compared to women with no history of early sexual abuse (Classen, Palesh, \& Aggarwal, 2005). We controlled for acute effects of revictimization by excluding individuals who experienced sexual abuse in the previous 3 months, but we did not check for revictimization after age 16; therefore, it is possible that some women in our CSA group experienced sexual revictimization as adults. These women may present with different (perhaps more impaired) sexual selfschemas so future studies would benefit from distinguishing between individuals abused only during childhood and those abused during childhood and adulthood. It is also worth noting that this study selected a relatively narrow group of women with a history of sexual abuse. Future studies that recruit women with a greater variety of sexual abuse histories (i.e., including also sexual abuse that occurred during adulthood) may be able to explain important nuances in the aspects of sexual abuse that are more closely associated with sexual arousal function and sexual satisfaction.

It is noteworthy that the CSA group showed a high prevalence of a variety of types of childhood maltreatment, including physical and emotional abuse and neglect. It is indeed the norm for different types of childhood adversities to co-occur, with a study estimating that approximately $85 \%$ of people who reported being molested during childhood also reporting a second or third type of childhood adversity, and $98 \%$ of people reporting rape during childhood also reporting a second or third type of childhood adversity; meaning that only $2 \%$ of the people who reported being raped during childhood only experienced rape (Kessler, Davis, \& Kendler, 1997). This high comorbidity of childhood maltreatment may affect the interpretation of our results since we are unable to distinguish the unique effects of sexual abuse on adult sexual functioning. Because of the recruitment protocol utilized in this study, it would be bias to analyze physical and emotional abuse and neglect separately since we have selected specifically individuals with a history of sexual abuse. Future studies will need to recruit women who experienced a variety of childhood maltreatment types to parcel out the effects of sexual abuse from other forms of childhood maltreatment.

In conclusion, this study provided evidence that sexual selfschemas predicted negative affect prior to exposure to sexual stimuli, which, in turn, predicted levels of sexual satisfaction. The lower sexual satisfaction of women with a history of CSA was partially explained by higher reports of negative affect prior to sexual stimuli. However, sexual self-schemas did not explain why women with a history of CSA experienced greater negative affect prior to sexual stimuli. It should be noted that the Embarrassed/Conservative schemas were not specific to women with a history of CSA, they appear to be related to the negative affect reported prior to sexual stimuli. Given the important role of negative affect on sexual satisfaction and function, and that we found only moderate effects in the relationship between Embarrassed/Conservative schemas and negative affect, future studies should focus on the etiology of negative affect prior to exposure to sexual stimuli. Future studies that can determine the mechanisms by which women with a history of CSA experience more negative affect prior to exposure to sexual stimuli may reveal additional cognitive dimensions that can be targeted in clinical treatment.

Acknowledgments This research was supported by Grant Number 1RO1HD051676 from the National Institute for Child Health and Human Development to Cindy M. Meston, by Grant Number F31 MH68165 from the National Institute of Mental Health to Alessandra Rellini and Cindy M. Meston, and by the McNeil Prevention and Community Psychology Fund to Alessandra Rellini. Its contents are solely the responsibility of the authors and do not necessarily represent the official views of the National Institute for Child Health and Human Development or the National Institute of Mental Health. The authors would like to thank Drs. Dianna Murray-Close and Keith Burt for their statistical consultation, and Carolyn Dundon, Samantha Elinson, Saiful Islam, and Sarah Roberts for their substantial contributions with data collection and the reduction of psychophysiological data.

Open Access This article is distributed under the terms of the Creative Commons Attribution Noncommercial License which permits any noncommercial use, distribution, and reproduction in any medium, provided the original author(s) and source are credited.

\section{References}

American Psychiatric Association. (2000). Diagnostic and statistical manual of mental disorders (4th ed., text revision). Washington, DC: Author.

Andersen, B. L., \& Cyranowski, J. M. (1994). Women's sexual selfschema. Journal of Personality and Social Psychology, 67, 10791100 .

Barlow, D. H. (1986). Causes of sexual dysfunction: The role of anxiety and cognitive interference. Journal of Consulting and Clinical Psychology, 54, 140-148.

Beck, A. T. (1996). Beyond belief: A theory of modes, personality and psychopathology. In P. M. Salkovskis (Ed.), Frontiers of cognitive therapy (pp. 1-25). New York: Guilford Press.

Beck, J. G., \& Barlow, D. H. (1986). The effects of anxiety and attentional focus on sexual responding. II. Cognitive and affective patterns in erectile dysfunction. Behaviour Research and Therapy, 24, 19-26.

Beck, J. G., Barlow, D. H., Sakheim, D. K., \& Abrahamson, D. J. (1987). Shock threat and sexual arousal: The role of selective attention, 
thought content, and affective states. Psychophysiology, 24, 165172.

Beggs, V. E., Calhoun, K. S., \& Wolchik, S. A. (1987). Sexual anxiety and female sexual arousal: A comparison of arousal during sexual anxiety stimuli and sexual pleasure stimuli. Archives of Sexual Behavior, 16, 311-319.

Bernstein, D. P., \& Fink, L. (1998). Childhood Trauma Questionnaire: A retrospective self-report manual. San Antonio, TX: The Psychological Corporation.

Bernstein, D., Fink, L., Handelsman, L., Foote, J., Lovejoy, M., \& Wenzel, K. (1994). Initial reliability and validity of a new retrospective measure of child abuse and neglect. American Journal of Psychiatry, 151, 1132-1136.

Carpenter, K. M., Andersen, B. L., Fowler, J. M., \& Maxwell, G. L. (2009). Sexual self schema as a moderator of sexual and psychological outcomes for gynecologic cancer survivors. Archives of Sexual Behavior, 38, 828-841.

Classen, C. C., Palesh, G. O., \& Aggarwal, R. (2005). Sexual revictimization: A review of the empirical literature. Trauma, Violence \& Abuse, 6, 103-129.

Cyranowski, J. M., \& Andersen, B. L. (1998). Schemas, sexuality, and romantic attachment. Journal of Personality and Social Psychology, 74, 1364-1379.

Dove, N. L., \& Weiderman, M. W. (2000). Cognitive distraction and women's sexual functioning. Journal of Sex and Marital Therapy, $26,67-78$.

Elliot, A. N., \& O'Donohue, W. T. (1997). The effects of anxiety and distraction on sexual arousal in a nonclinical sample of heterosexual women. Archives of Sexual Behavior, 26, 607-624.

Finkelhor, D., Hotaling, G. T., Lewis, I. A., \& Smith, C. (1989). Sexual abuse and its relationship to later sexual satisfaction, marital status, religion, and attitudes. Journal of Interpersonal Violence, 4, 379-399.

Gibb, B.E., \& Alloy, L. B. (2006). A prospective test of the hopelessness theory of depression in children. Journal of Clinical Child and Adolescent Psychology, 35, 264-274.

Heiman, J. R. (1980). Female sexual response patterns: Interactions of physiological, affective, and contextual cues. Archives of General Psychiatry, 37, 1311-1316.

Heiman, J. R. (2002). Sexual dysfunction: Overview of prevalence, etiological factors, and treatments. Journal of Sex Research, 39, 73-78.

Heiman, J. R., \& Rowland, D. L. (1983). Affective and physiological sexual response patterns: The effects of instructions on sexual functional and dysfunctional men. Journal of Psychosomatic Research, 27, 105-116.

Hill, D. B. (2007). Differences and similarities in men's and women's sexual self-schemas. Journal of Sex Research, 44, 135-144.

Kessler, R. C., Davis, C. G., \& Kendler, K. S. (1997). Childhood adversity and adult psychiatric disorder in the US National Comorbidity Survey. Psychological Medicine, 27, 1101-1119.

Kihlstrom, J. F., \& Cantor, N. (1983). Mental representations of the self. In L. Berkowitz (Ed.), Advances in experimental social psychology (Vol. 17, pp. 1-47). New York: Academic.

Koukounas, E., \& McCabe, M. P. (2001). Sexual and emotional variables influencing sexual response to erotica: A psychophysiological investigation. Archives of Sexual Behavior, 30, 393-408.

Kuffel, S. W., \& Heiman, J. R. (2006). Effects of depressive symptoms and experimentally adopted schemas on sexual arousal and affect in sexually healthy women. Archives of Sexual Behavior, 35, 163-177.

Laan, E., \& Everaerd, W. (1995). Determinants of female sexual arousal: Psychophysiological theory and data. Annual Review of Sex Research, 6, 32-76.

Laan, E., Everaerd, W., van Aanhold, M. T., \& Rebel, M. (1993). Performance demand and sexual arousal in women. Behaviour Research and Therapy, 31, 25-35.
Leiblum, S., \& Wiegel, M. (2002). Psychotherapeutic interventions for treating female sexual dysfunction. World Journal of Urology, 20, 127-136.

Leonard, L. M., \& Follette, V. M. (2002). Sexual functioning in women reporting a history of child sexual abuse: Review of the literature and clinical implications. Annual Review of Sex Research, 13, $346-388$

Loeb, T. B., Williams, J. K., Carmona, J. V., Rivkin, I., Wyatt, G. E., Chin, D., et al. (2002). Child sexual abuse: Associations with the sexual functioning of adolescents and adults. Annual Review of Sex Research, 13, 307-345.

Lumley, M. N., \& Harkness, K.L. (2007). Specificity in the relations among childhood adversity, early maladaptive schemas, and symptom profiles in adolescent depression. Cognitive Therapy and Research, 31, 639-657.

MacKinnon, D. P., \& Dwyer, J. H. (1993). Estimating mediated effects in prevention studies. Evaluation Review, 17, 144-158.

MacKinnon, D. P., Fairchild, A. J., \& Fritz, M. S. (2007). Mediation analysis. Annual Review of Psychology, 58, 93-614.

Markus, H. (1990). On splitting the universe. Psychological Science, 1, $181-185$

Markus, H., \& Zajonc, R. B. (1985). The cognitive perspective in social psychology. In G. Lindzey \& E. Aronson (Eds.), Handbook of social psychology (Vol. 3, pp. 137-230). Hillsdale, NJ: Erlbaum.

Meisler, A. W., \& Carey, M.P.(1991). Depressed affect and male sexual arousal. Archives of Sexual Behavior, 20, 541-554.

Meston, C. M. (2003). Validation of the Female Sexual Function Index (FSFI) in women with female orgasmic disorder and in women with hypoactive sexual desire disorder. Journal of Sex and Marital Therapy, 29, 39-46.

Meston, C. M., Rellini, A. H., \& Heiman, J. R. (2006). Women's history of sexual abuse, their sexuality, and sexual self-schemas. Journal of Consulting and Clinical Psychology, 74, 229-236.

Meston, C. M., \& Trapnell, P. D. (2005). Development and validation of a five factor sexual satisfaction and distress scale: The Sexual Satisfaction Scale for Women (SSS-W). Journal of Sexual Medicine, 2, 66-81.

Mitchell, W. B., DiBartolo, P. M., Brown, T. A., \& Barlow, D. H. (1998). Effects of positive and negative mood on sexual arousal in sexually functional males. Archives of Sexual Behavior, 27, 197-207.

Nobre, P. J., \& Pinto-Gouveia, J. (2008). Cognitions, emotions, and sexual responses: Analysis of the relationship among automatic thoughts, emotional responses, and sexual arousal. Archives of Sexual Behavior, 37, 652-661.

Palace, E. M., \& Gorzalka, B. B. (1990). The enhancing effects of anxiety on arousal in sexually dysfunctional and functional women. Journal of Abnormal Psychology, 99, 403-411.

Putnam, F. W. (1990). Disturbances of "self" in victims of childhood sexual abuse. In R. P. Kluft (Ed.), Incest-related syndromes of adult psychopathology (pp. 113-131). London: American Psychiatric Press.

Rellini, A. H. (2008). Review of the empirical evidence for a theoretical model to understand the sexual problems of women with a history of CSA. Journal of Sexual Medicine, 5, 31-46.

Rellini, A. H., McCall, K. M., Randall, P. K., \& Meston, C. M. (2005). The relationship between women's subjective and physiological sexual arousal. Psychophysiology, 42, 116-124.

Rellini, A. H., \& Meston, C. M. (2006). Psychophysiological sexual arousal in women with a history of childhood sexual abuse. Journal of Sex and Marital Therapy, 32, 5-22.

Rellini, A. H., \& Meston, C. M. (2007). Sexual function and satisfaction in adults based on the definition of child sexual abuse. Journal of Sexual Medicine, 4, 1312-1321.

Reynolds, C. F., Frank, E., \& Thase, M. E. (1988). Assessment of sexual function in depressed, impotent, and healthy men: Factor analysis of a 
Brief Sexual Function Questionnaire for Men. Psychiatry Research, 24, 231-250.

Rosen, R., Brown, C., Heiman, J., Leiblum, S., Meston, C., Shabsigh, R., et al. (2000). The Female Sexual Function Index (FSFI): A multidimensional self-report instrument for the assessment of female sexual function. Journal of Sex and Marital Therapy, 26, 191-208.

Schacht, R. L., George, W. H., Heiman, J. R., Norris, J., Davis, K. C., Stoner, S. A., et al. (2007). Effects of alcohol intoxication and instructional set on women's sexual arousal vary based on sexual abuse history. Archives of Sexual Behavior, 36, 655-665.

Sintchak, G., \& Geer, J. H. (1975). A vaginal plethysmograph system. Psychophysiology, 12, 113-115.

Sobel, M. E. (1982). Asymptotic confidence intervals for indirect effects in structural equation models. In S. Leinhardt (Ed.), Sociological methodology (pp. 290-312). Washington, DC: American Sociological Association.

Stuewig, J., \& McCloskey, L. A. (2005). The relation of child maltreatment to shame and guilt among adolescents: Psychological routes to depression and delinquency. Child Maltreatment, 10, $324-336$.
Taylor, J. F., Rosen, R. C., \& Leiblum, S. R. (1994). Self report assessment of female sexual function: Psychometric evaluation of the Brief Index of Sexual Functioning of Women. Archives of Sexual Behavior, 23, 627-643.

Watson, D. (2000). Mood and temperament. New York: Guilford Press. Watson, D., Clark, L. A., \& Tellegen, A. (1988). Development and validation of brief measures of positive and negative affect: The PANAS scales. Journal of Personality and Social Psychology, 54, 1063-1070.

Wiegel, M., Scepkowski, L., \& Barlow, D. H. (2007). Cognitiveaffective processes in sexual arousal and sexual dysfunction. In E. Janssen (Ed.), The psychophysiology of sex (pp. 143-165). Bloomington: Indiana University Press.

Wincze, J. P., \& Carey, M. P. (2001). Sexual dysfunction: A guide for assessment and treatment (2nd ed.). New York: Guilford Press.

Wincze, J. P., Hoon, E. F., \& Hoon, P. W. (1977). Multiple measure analysis of women experiencing low sexual arousal. Behaviour Research and Therapy, 16, 43-49. 